\title{
Rapid identification and characterization of genetic loci for defective kernel in bread wheat
}

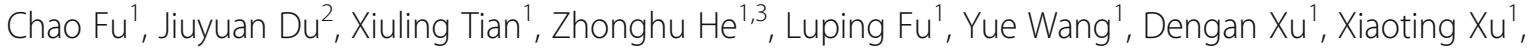 \\ Xianchun Xia ${ }^{1}$, Yan Zhang ${ }^{1 *}$ and Shuanghe Cao ${ }^{1 *}$
}

\begin{abstract}
Background: Wheat is a momentous crop and feeds billions of people in the world. The improvement of wheat yield is very important to ensure world food security. Normal development of grain is the essential guarantee for wheat yield formation. The genetic study of grain phenotype and identification of key genes for grain filling are of great significance upon dissecting the molecular mechanism of wheat grain morphogenesis and yield potential.

Results: Here we identified a pair of defective kernel (Dek) isogenic lines, BL31 and BL33, with plump and shrunken mature grains, respectively, and constructed a genetic population from the BL31/BL33 cross. Ten chromosomes had higher frequency of polymorphic single nucleotide polymorphism (SNP) markers between BL31 and BL33 using Wheat660K chip. Totally 783 simple sequence repeat (SSR) markers were chosen from the above chromosomes and 15 of these were integrated into two linkage groups using the genetic population. Genetic mapping identified three QTL, QDek.caas-3BS.1, QDek.caas-3BS.2 and QDek.caas-4AL, explaining 14.78-18.17\%, 16.61-21.83\% and 19.08$28.19 \%$ of phenotypic variances, respectively. Additionally, five polymorphic SNPs from Wheat660K were successfully converted into cleaved amplified polymorphic sequence (CAPS) markers and enriched the target regions of the above QTL. Biochemical analyses revealed that BL33 has significantly higher grain sucrose contents at filling stages and lower mature grain starch contents than BL31, indicating that the Dek QTL may be involved in carbohydrate metabolism. As such, the candidate genes for each QTL were predicated according to International Wheat Genome Sequence Consortium (IWGSC) RefSeq v1.0.
\end{abstract}

Conclusions: Three major QTL for Dek were identified and their causal genes were predicted, laying a foundation to conduct fine mapping and dissect the regulatory mechanism underlying Dek trait in wheat.

Keywords: Defective kernel, Grain filling, Rapid QTL mapping, SNP array, Triticum aestivem L

\section{Background}

Wheat (Triticum aestivum L.) supplies staple food for more than $35 \%$ of population worldwide (http://www.wheatinitiative.org/sites/default/files/attached_file/wheat_facts. pdf). The increase of population, decline of cultivated land and frequent occurrence of extreme climate posed large challenges to meet the consumption of wheat. Identification and mining of genetic loci for grain yield is important to improve the yield potential in wheat. Yield is determined by

\footnotetext{
* Correspondence: zhangyan07@caas.cn; caoshuanghe@caas.cn 'Institute of Crop Sciences, National Wheat Improvement Center, Chinese Academy of Agricultural Sciences, Beijing 100081, China Full list of author information is available at the end of the article
}

multiple components, such as grain weight and size. Grain plumpness is an important parameter affecting grain weight and morphological quality [1]. Many genetic loci controlling grain weight have been identified in previous studies [2], whereas genetic analyses on grain plumpness associated with grain filling is rarely reported in wheat. The defective kernel (Dek) mutants in gramineous crops are directly reflected in grain plumpness and usually characterized by shriveled kernel and low grain weight. Thus, identification of genetic loci for Dek is important to improve yield potential and appearance quality. Quite a few Dek mutants were identified in maize [3]. Of them, the genes of dek1, dek35, dek38 and dek39 have been isolated, and all involved in the

(c) The Author(s). 2019 Open Access This article is distributed under the terms of the Creative Commons Attribution 4.0 International License (http://creativecommons.org/licenses/by/4.0/), which permits unrestricted use, distribution, and reproduction in any medium, provided you give appropriate credit to the original author(s) and the source, provide a link to the Creative Commons license, and indicate if changes were made. The Creative Commons Public Domain Dedication waiver (http://creativecommons.org/publicdomain/zero/1.0/) applies to the data made available in this article, unless otherwise stated. 
synthesis and accumulation of starches and/or proteins in the endosperm [4-7]. Many Dek mutants have been generated in wheat, but their genetic information remains largely unknown. SSR markers are characterized with high polymorphisms, good repeatability, co-dominance, and distribution throughout whole genome, and favored for QTL mapping and molecular breeding in wheat [8-10]. With rapid advancements in sequencing technology, highthroughput sequencing or chip-based SNP genotyping platforms have been used for QTL mapping [11-14]. Additionally, the wheat reference genome was assembled and released recently [15]. The availability of high-quality wheat genome data and high-throughput SNP genotyping systems is very important to improve wheat genetics and breeding. We identified a Dek line in a spontaneous variation population and found that its kernels are severely shrunken and sunken. The objective of this study was to rapidly achieve QTL mapping and causal gene prediction for Dek through multiple genotyping systems, wheat reference genome and biochemical analysis.

\section{Results}

\section{Phenotypic analysis}

The kernels of BL31 were normal and plump, whereas the Dek rate of BL33 is 100\% in all environments (Additional file 1). The Dek rates in $F_{2}$ and $F_{2: 3}$ populations were highly correlated among three environments with correlation coefficients ranging from 0.70 to 0.92 (Table 1). Variance analyses showed that both genotypes $(P<0.001)$ and environments $(P<0.05)$ had significant effects on the target trait (Table 2 ). The genotype $\times$ environment interactions did not reach a statistical significance $(P>0.05)$ (Table 2) and the broad-sense heritability of Dek rate in $F_{2: 3}$ across two environments was 0.95 , denoting that the Dek rate was mainly determined by genotypes. The Dek rates in the populations showed continuous distribution (Fig. 1, a-d), indicating that Dek is controlled by multiple genetic loci. Additionally, the frequency graph underpinning the phenotypes is abnormal distribution with the "valley - peak" pattern, suggesting the presence of major QTL controlling Dek in the populations [16].

Table 1 Statistical analysis of Dek rates for $F_{2}$ and $F_{2: 3}$ populations across three environments

\begin{tabular}{lllcc}
\hline Environment & Range & $\begin{array}{l}\text { Mean } \pm \\
\text { SD }\end{array}$ & \multicolumn{2}{c}{ Correlation coefficient } \\
\cline { 4 - 5 } & & & Gaoyi 2018 & Xinxiang 2018 \\
\hline Gaoyi 2017 & $0 \sim 1.00$ & $0.33 \pm 0.32$ & $0.70^{* *}$ & $0.73^{* *}$ \\
Gaoyi 2018 & $0 \sim 1.00$ & $0.24 \pm 0.32$ & & $0.92^{* *}$ \\
Xinxiang 2018 $^{\mathrm{d}}$ & $0 \sim 1.00$ & $0.22 \pm 0.29$ & & \\
\hline
\end{tabular}

a SD, standard deviation. ${ }^{b}$ Gaoyi 2017, Gaoyi, 2016-2017 cropping season. ${ }^{c}$ Gaoyi 2018, Gaoyi, 2017-2018 cropping season. ${ }^{d}$ Xinxiang 2018, Xinxiang, 2017-2018 cropping season. ${ }^{* *}$ indicates significance levels at $P<0.01$

\section{Chromosomal locations of Dek loci and construction of linkage maps}

Theoretically, the more polymorphic markers between isogenic lines one chromosome contains, the higher probability that it harbors QTL for target traits. To rapidly identify the chromosomal locations of Dek QTL, we genotyped the isogenic lines, BL31 and BL33, with contrasting grain phenotypes using the Wheat660K SNP array. Ten chromosomes, 1A, 1B, 2A, 3A, 3B, 4A, 4B, $5 \mathrm{~A}, 6 \mathrm{~B}$ and $7 \mathrm{~B}$, were considered to be potential target chromosomes with Dek QTL based on the frequency distributions of polymorphic SNPs (Additional file 2). To further verify the chromosomal location of Dek QTL, SSR markers from the above target chromosomes were selected to construct genetic map. Totally 783 SSR markers on these chromosomes were selected to construct linkage map for QTL analysis (Additional file 3) [8, 17-23]. Of them, 118 SSR markers displayed polymorphisms between two parents, while only 35 SSRs in chromosomes 3B (13), 4A (19) and 5A (3), showed consistent polymorphisms between two groups of lines with contrasting phenotypes, i.e. 40 defective kernel and 40 normal kernel lines, respectively. Subsequently, the polymorphic SSR markers were used to genotype the entire $\mathrm{F}_{2}$ population, and two linkage groups were constructed in chromosomes $3 \mathrm{~B}$ and $4 \mathrm{~A}$, including 6 and 9 SSR markers, respectively (Fig. 2).

\section{QTL mapping}

Based on the resultant linkage groups above and Dek rates in the genetic populations, we performed QTL mapping for Dek traits; three QTL were detected on chromosomes 3B and 4A, designated as QDek.caas-3BS.1, QDek.caas-3BS.2 and QDek.caas-4AL, respectively (Table 3; Fig. 2). QDek.caas-3BS.1 was flanked by Xbarc133 and Xcfd79 spanning a genetic interval of $6.77 \mathrm{cM}$; QDek.caas-3BS.2 was located between Xcfd79 and Xwmc808 in an interval of $35.03 \mathrm{cM}$; QDek.caas-4AL was mapped between Xwmc500 and Xgpw3079 in a genetic interval of 15.17 cM (Fig. 2). Based on the preliminary mapping above, the physical regions of QDek.caas-3BS.1, QDek.caas-3BS.2 and QDek.caas-4AL can be defined by their flanking markers according to IWGSC reference genome RefSeq v1.0 (http://plants.ensembl.org/ Triticum_aestivum/Info/Index) [15]. The physical intervals of QDek.caas-3BS.1, QDek.caas-3BS.2 and QDek.caas-4AL are $7.60-18.84 \mathrm{Mb}, 18.84-32.75 \mathrm{Mb}$ in chromosomes 3BS and 704.34-710.05 Mb in chromosome 4AL. Genetic analyses showed that QDek.caas-3BS.1, QDek.caas-3BS.2 and QDek.caas-4AL could explain 14.78 to $18.17 \%, 16.61$ to $21.83 \%$ and 19.08 to $28.19 \%$ of the phenotypic variances, respectively (Table 3). Each of these QTL determined more than $10 \%$ phenotypic variation of Dek in each environment, indicating that they are major QTL, consistent with the "peak - valley" distribution pattern of Dek rate in genetic 
Table 2 Analysis of variance of Dek rates for $F_{2: 3}$ lines derived from BL33/BL31

\begin{tabular}{lllll}
\hline Source of variation & Degrees of freedom & Sum of square & Mean square & $F$ value \\
\hline Environment & 1 & 0.097 & 0.097 & $0.364^{*}$ \\
Genotype & 145 & 51.76 & $20.68^{* * *}$ \\
Genotype $\times$ Environment & 145 & 2.21 & 0.015 \\
Error & 290 & 5.01 & 0.017 \\
\hline
\end{tabular}

${ }^{*}$ indicates significance levels at $P<0.05 .{ }^{* * *}$ indicates significance levels at $P<0.001$

populations. Additionally, all the alleles governing the formation of Dek came from BL33.

\section{Marker enrichment in the target regions of QTL}

To further narrow down the genetic intervals of target QTL, the polymorphic SNPs identified from the Wheat660K chips were selected according to the above physical regions defined by QTL preliminary mapping. Totally 60 polymorphic SNPs were used to develop sitespecific CAPS markers and five of these, $A X-109027972$, $A X-109516011, A X-110042240, A X-109301653$ and $A X-$ 108996126, were successfully mapped in the linkage groups (Additional file 4; Additional file 5; Additional file 6). Subsequently, QDek.caas-3BS.2 and QDek.caas-4AL were narrowed to the genetic intervals of $14.01 \mathrm{cM}$ and $13.18 \mathrm{cM}$, respectively (Table 3; Fig. 2). Accordingly, the physical intervals of QDek.caas-3BS.2 and QDek.caas-4AL are reduced to $1.16 \mathrm{Mb}$ and $1.13 \mathrm{Mb}$, respectively (Table 3). Although the polymorphic loci $A X-108996126$ and $A X$ 110042240 did not further diminish the genetic interval of QDek.caas-3BS.1 and QDek.caas-4AL, they saturated the target regions (Fig. 2). Dek trait displays abnormal development of grain and thus it is usually considered to affect grain weight. To verify this case, we analyzed the effect of the resultant Dek QTL on thousand grain weight (TGW). Compared with BL31, the TGW of BL33 significantly decreased 24.12-28.81\% (Additional file 7). QTL analyses based on the linkage maps above showed that only one QTL for TGW, designated as QTGW.caas-3BS, was detected between $X c f d 79$ and $A X-109027972$, explaining 19.18 to $23.94 \%$ of phenotypic variances (Additional file 8 ). QTGW.caas-3BS overlapped QDek.caas-3BS.2 on the linkage map, suggesting that the Dek QTL QDek.caas-3BS.2 may affect TGW. No significant effect on TGW was
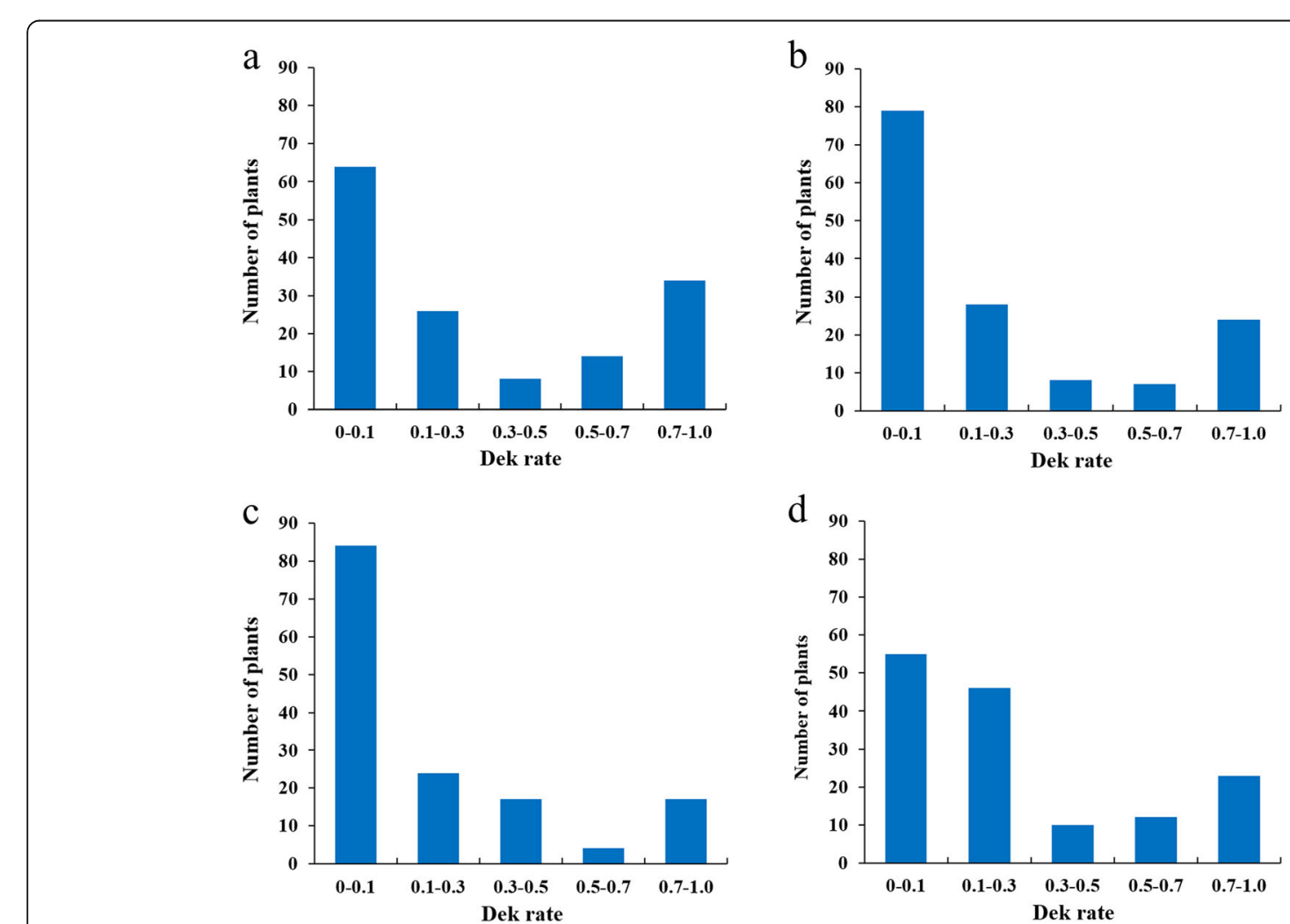

Fig. 1 The frequency distributions of Dek rates in Gaoyi 2016-2017 cropping season (a), Gaoyi 2017-2018 cropping season (b), Xinxiang 20172018 cropping season (c), and the average data across three environments (d) 


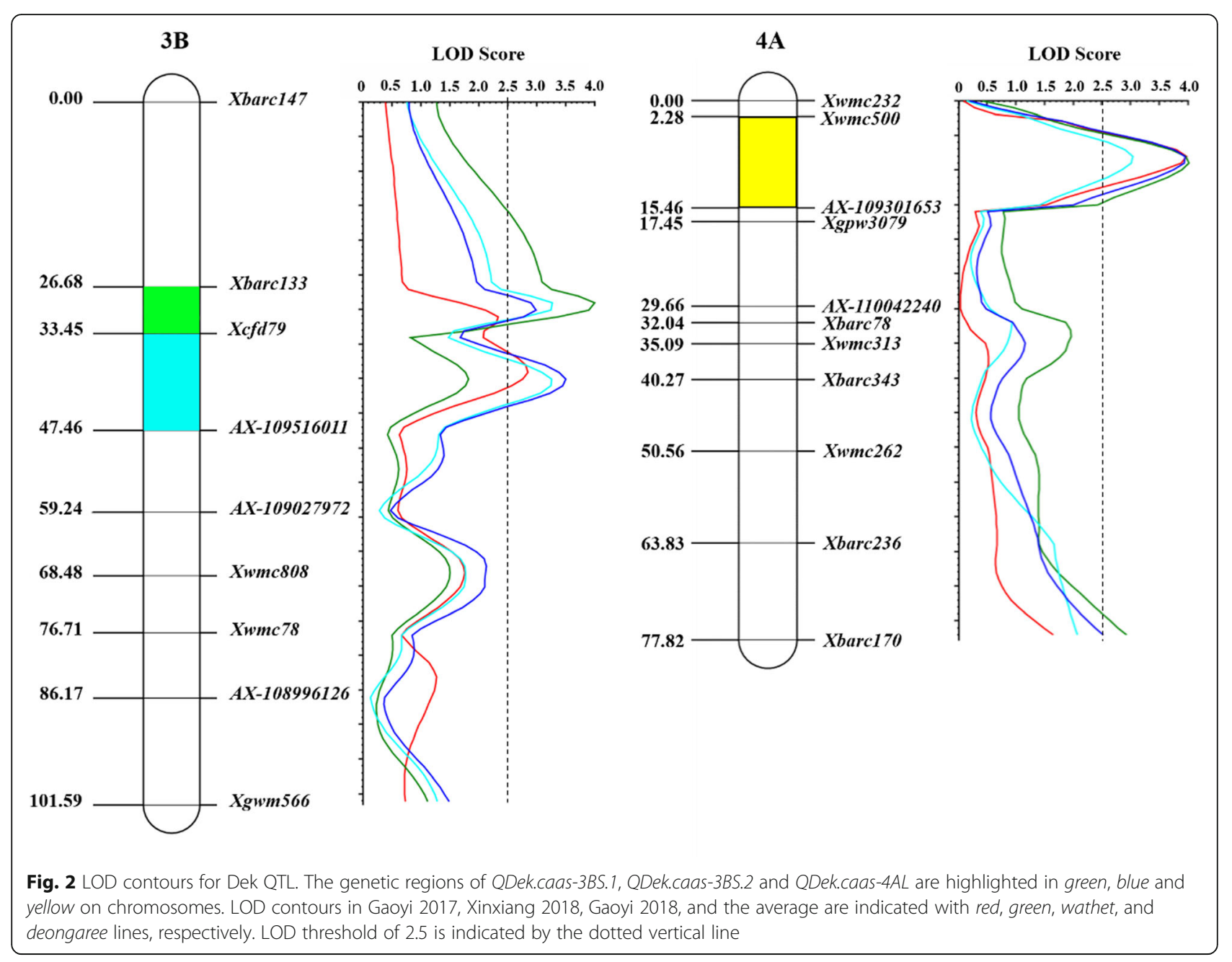

Table 3 QTL for Dek trait detected in the $F_{2}$ and $F_{2: 3}$ populations across different environments

\begin{tabular}{|c|c|c|c|c|c|c|}
\hline Environment & QTL & $\begin{array}{l}\text { Flanking } \\
\text { marker }\end{array}$ & $\begin{array}{l}\text { Physical } \\
\text { interval } \\
(\mathrm{Mb})^{\mathrm{a}}\end{array}$ & LOD & $\mathrm{Add}^{\mathrm{b}}$ & PVE $(\%)^{c}$ \\
\hline \multirow[t]{2}{*}{ Gaoyi $2017^{d}$} & QDek.caas-3BS.2 & Xcfd79-AX-109516011 & $18.84-20.00$ & 2.84 & 0.13 & 16.61 \\
\hline & QDek.caas-4AL & Xwmc500-AX-109301653 & 704.34-705.47 & 3.98 & 0.06 & 28.19 \\
\hline \multirow[t]{2}{*}{ Xinxiang $2018^{e}$} & QDek.caas-3BS.1 & Xbarc133-Xcfd79 & $7.60-18.84$ & 4.00 & 0.14 & 18.17 \\
\hline & QDek.caas-4AL & Xwmc500-AX-109301653 & 704.34-705.47 & 4.01 & 0.12 & 22.60 \\
\hline \multirow[t]{3}{*}{ Gaoyi $2018^{f}$} & QDek.caas-3BS.1 & Xbarc133-Xcfd79 & $7.60-18.84$ & 3.26 & 0.14 & 16.39 \\
\hline & QDek.caas-3BS.2 & Xcfd79-AX-109516011 & $18.84-20.00$ & 3.25 & 0.17 & 21.83 \\
\hline & QDek.caas-4AL & Xwmc500-AX-109301653 & 704.34-705.47 & 3.03 & 0.09 & 19.08 \\
\hline \multirow[t]{3}{*}{ Average } & QDek.caas-3BS.1 & Xbarc133-Xcfd79 & $7.60-18.84$ & 2.98 & 0.11 & 14.78 \\
\hline & QDek.caas-3BS.2 & Xcfd79-AX-109516011 & $18.84-20.00$ & 3.49 & 0.13 & 21.80 \\
\hline & QDek.caas-4AL & Xwmc500-AX-109301653 & 704.34-705.47 & 3.95 & 0.09 & 23.38 \\
\hline
\end{tabular}

${ }^{a}$ Physical interval defined by physical positions of flanking markers according to IWGSC RefSeq v1.0. ${ }^{\mathrm{b}}$ Add, additive effect; positive values indicate an increasing effect from BL33. ${ }^{c}$ PVE, phenotypic variance explained. ${ }^{d}$ Gaoyi 2017, Gaoyi, 2016-2017 cropping season. ${ }^{e}$ Gaoyi 2018, Gaoyi, $2017-2018$ cropping season. ${ }^{f}$ Xinxiang 2018, Xinxiang, 2017-2018 cropping season 
detected in the genetic intervals of the other two QTL, QDek.caas-3BS.1 and QDek.caas-4AL.

\section{Predication of candidate genes for the Dek QTL}

The cloned dek genes in maize are involved in the grain growth and development, especially the synthesis and storage of starches and/or proteins in the endosperm [4-7]. Our biochemical analysis also showed that the mature grain starch contents of BL33 were significantly lower than those of BL31 (Additional file 9). In contrast, BL33 had higher sucrose contents and slower sucrose reduction across the filling stages than BL31 (Additional file 10). Accordingly, we assumed that the causal genes for the three QTL are involved in carbohydrate metabolism. In this study, three major QTL was mapped into the genetic intervals of less than $15 \mathrm{cM}$. The physical region of QDek.caas-3BS.1 is $11.24 \mathrm{Mb}$, whereas those of QDek.caas-3BS.2 and QDeg.caas-4AL are only 1.16 and $1.13 \mathrm{Mb}$, respectively, according to IWGSC RefSeq v1.0 (Table 3). Totally 233 genes were present in the physical intervals of the three QTL (Additional file 11). We found nine genes (Gene ID: TraesCS3B01G025200, TraesCS3B01G028100, TraesCS3 B01G028200, TraesCS3B01G028300, TraesCS3B01G0 28500, TraesCS3B01G028700, TraesCS3B01G039100, Tra esCS3B01G039800, and TraesCS4A01G446700) involving carbohydrate metabolism or grain development in or near the target regions of the Dek QTLs (Additional file 11). To further identify candidate genes for the Dek QTL, the spatio-temporal expression patterns of the nine genes were analyzed using the WheatExp (https://wheat.pw.usda.gov/ WheatExp/). Of these, four genes, TraesCS3B01G025200, TraesCS3B01G028700, TraesCS3B01G039800, and TraesCS4A01G446700, had an observable expression during grain development (Additional file 12) [24]. Based on functional annotation in IWGSC RefSeq v1.0, the four genes encoded fructose-bisphosphate aldolase (Fba), $\beta$-fructofuranosidase, abscisic acid-deficient 4 (ABA4), and sucrose synthase (Sus), respectively, tentatively designated as TaFba-3B, TaBff-3B, TaABA4-3B, and TaSus-4A. Their expression patterns were confirmed in the immature grains harvested at three different stages $(5,15$, and $25 \mathrm{DAF})$ using qPCR (Additional file 13). The results showed that all of the above genes expressed in grain and had the lowest expression level at 5 DAF. TaBff-3B and TaSus- $4 A$ was mainly detected at 15 DAF, while TaFba-3B and TaABA4-3B had the highest expression activity in 25-DAF grains.

\section{Discussion}

A rapid and cost-effective QTL mapping strategy through multiple genotyping systems in combination with wheat reference genome

With rapid advances in sequencing technology, more and more wheat varieties were sequenced and a large number of SNPs have been identified [25-27]. Consequently, a few sequencing-based and chip-based SNP genotyping platforms have been developed and used for genetic analyses in wheat [28-30]. Some studies have achieved fine mapping of QTL using SNP chips [31-34]. The chip-based SNP arrays are high-throughput and effective for genetic analyses, but the chips are very expensive. In this study, to rapidly and cost-effectively map QTL for Dek, we only genotyped the isogenic parental lines with contrasting phenotypes using the Wheat660K chip. Firstly, the target chromosomes potentially harboring the QTL for Dek were determined according to the frequency distributions of the polymorphic SNPs. Secondly, it is necessary to construct linkage map for QTL analysis through genotyping genetic populations. However, it is well-known that successful conversion of SNP to the flexible markers, such as KASP and CAPS, is laborious and time-consuming, especially in the hexaploid wheat with three homoeologous subgenomes. Considering high polymorphisms, good repeatability, co-dominance, and distribution throughout whole genome of SSR markers, we harvested the handy SSR markers in the candidate chromosomes above and rapidly constructed linkage maps $[8,35]$. Thirdly, we performed genetic mapping for Dek QTL and defined their physical intervals according to IWGSC RefSeq v1.0. Finally, we successfully converted some of the polymorphic SNPs in the physical target regions to CAPS markers and narrowed down the genetic regions of the QTL. In all, we set up a rapid and efficient strategy for QTL mapping through combining high-throughput SNP arrays with cost-effective and flexible SSR markers as well as wheat reference genome (Fig. 3). Of course, a genome-wide linkage map is necessary to find the QTL in all chromosomes. In the future, we intend to construct a recombinant inbred line (RIL) population and achieve genome-wide identification for Dek QTL.

\section{Comparisons of the Dek QTL with previously reported Dek-related genetic loci}

The comparisons of genetic loci for Dek-related traits can not only improve the understanding of genetic effects of the resultant QTL in the present study but also supply with genetic information for QTL fine mapping. QDek.caas-3BS.1 is linked with Xbarc133 (Table 3; Fig. 2). Two QTL, QGfrmax.nfcri-3B and QGfd.nfcri-3B, controlling grain filling rate (GFR) and grain filling duration (GFD), respectively, were linked to Xgwm533 with a physical distance of $1 \mathrm{Mb}$ from Xbarc133 [37]. QTgw.nfcri-3B for TGW was also linked to Xgwm533 [37]. QDek.caas-3BS.1 and QDek.caas-3BS.2 were identified in adjacent regions and both are linked to Xcfd79 (Table 3; Fig. 2). A QTL for TGW linked with $X c f d 79$ was detected by Groos et al. [38]. Additionally, QMswscf.acs-3B.1 for water soluble carbohydrates (WSC) of main stem at the early anthesis stage, were linked to Xcfd79 [39]. QDek.caas-4AL is 


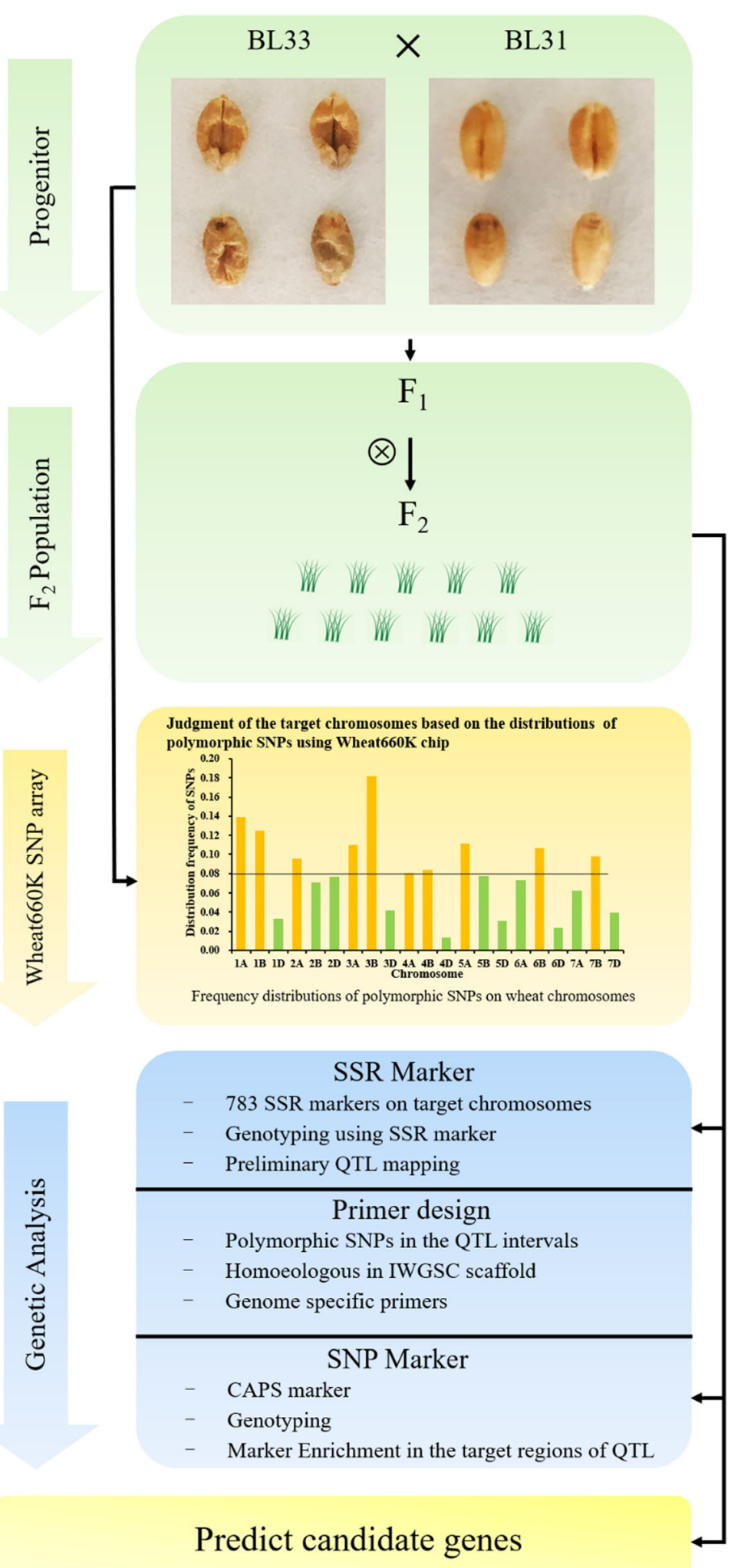

Fig. 3 The workflow of rapid and cost-effective QTL mapping for Dek trait. The production of our Fig. 3 referred to the frame of Fig. 1 in RamirezGonzalez et al. [36] 
flanked by Xwmc500 and $A X-109301653$ (Table 3). The physical distance between QDek.caas-4AL and $W x-B 1$ is $16 \mathrm{Mb}$ approximately. So far, many QTL for the contents of starch, protein and amylose and amylopectin, and grain yield have been reported on chromosome 4AL close to $W x-B 1$ [40-47]. QGsc4A, a QTL for grain starch contents, is closely linked to $W x-B 1$ [42]. Qftsc $4 A$ for total grain starch content and $Q$ fams $4 A$ for grain amylose content were also linked to $W x-B 1$ [46]. To identify the genetic relationship between QDek.caas-4AL and $W x-B 1$, the gene-specific functional marker developed by Nakamura et al. [48] was used to genotype the parents, BL31 and BL33. The result showed that no polymorphism was detected between them (Additional file 14), suggesting that $W x$ - $B 1$ had little effect on Dek trait.

\section{Candidate genes analysis}

Two candidate genes, TaFba-3B and TaBff-3B, were detected in QDek.caas-3BS.1 region, and encoded Fba and $\beta$-fructofuranosidases, respectively (Table 4). Fba is one of the nonregulated enzymes comprising the Calvin cycle and controlling carbon fixation and starch synthesis in photosynthetic organs, which can catalyze reversible aldol condensation reaction of dihydroxy acetone phosphate and 3-phosphate glyceraldehyde yielding fructose-1,6-diphosphate $[49,50]$. $\beta$-fructofuranosidase is a key enzyme for synthesis, metabolism and transportation of sucrose and starch, and can irreversibly catalyze sucrose hydrolysis to glucose and fructose [51, 52]. TaFba-3B and TaBff-3B probably regulate carbohydrate metabolism and consequently affect Dek phenotype. TaABA4-3B was predicted as a candidate gene for QDek.caas-3BS.2. ABA4 functions in neoxanthin synthesis [53]. Neoxanthin is necessary for maintaining the integrity of the light harvesting chlorophyll and photosynthetic function of leaves, involving the metabolism of photosynthetic product $[54,55]$. TaSus- $4 A$ may be the causal gene of QDek.caas-4AL. Sus deficiency led to the shrunken-1 mutations in maize [56]. Association analyses showed that the two sucrose synthase genes, TaSus 1 and TaSus 2 on wheat homoeologous chromosome groups 7 and 2, respectively, had significant effects on TGW $[57,58]$. In near future, we will identify polymorphic sites of these candidate genes to conduct fine mapping for the target QTL and further determine the causal genes for Dek through wheat transformation experiments.

\section{Conclusions}

In the study, we detected three DEK QTLs, QDek.caas3BS.1, QDek.caas-3BS.2 and QDek.caas-4AL, each of which could explain more than $10 \%$ phenotypic variation. Additionally, their candidate genes, TaFba-3B, TaBff-3B, TaABA4-3B, and TaSus-4A, were predicated according to biochemical analysis and IWGSC RefSeq v1.0 annotation. These findings provide important information for QTL fine mapping and molecular mechanistic dissection underlying Dek in wheat.

\section{Methods}

\section{Plant materials}

A number of Dek lines were generated in Wheat Research Institute, Gansu Academy of Agricultural Science (GAAS). Through multi-generation self-crossing, we identified a pair of isogenic lines, BL31 and BL33, with plump and shrunken mature grains, respectively. Genetic populations comprising $146 \mathrm{~F}_{2}$ plants and their corresponding $\mathrm{F}_{2: 3}$ lines from the cross between BL31 and BL33 were created for QTL mapping.

\section{Field trials and phenotype evaluation}

The $F_{2}$ population and parents were planted at Gaoyi $\left(37^{\circ} 33^{\prime} \mathrm{N}, 114^{\circ} 26^{\prime} \mathrm{E}\right)$ in Hebei province during the $2016-$ 2017 cropping season. The $\mathrm{F}_{2: 3}$ lines were sown at Xinxiang $\left(34^{\circ} 53^{\prime} \mathrm{N}, 113^{\circ} 23^{\prime} \mathrm{E}\right)$ in Henan province and Gaoyi during the 2017-2018 cropping season. Field trials were arranged in randomized complete blocks with two replicates at each location. Each plot comprised a single $2 \mathrm{~m}$ row with 20 plants and spaced $30 \mathrm{~cm}$ apart. Grains were harvested in individual plants or lines. Grain phenotype of $F_{2}$ plants was scored using the percentages of defective kernels (defined as Dek rate) in single plants. For $\mathrm{F}_{2: 3}$ lines, ten representative plants in each line were selected from the middle part of each plot to figure out Dek rate, then the average Dek rate of each plot was used for subsequent statistical and genetic analysis.

\section{Preliminary mapping of QTL on target chromosomes using SNP chip}

High-quality genomic DNA was extracted from fresh leaves following Guillemaut and Laurence [59]. The Wheat660K SNP array containing 630,517 SNPs, a highthroughput genotyping tool developed by Institute of Crop Sciences, Chinese Academy of Agricultural Sciences

Table 4 Candidate genes of Dek QTLs

\begin{tabular}{llll}
\hline QTL & Temporary name & Gene ID & Candidate gene \\
\hline QDek.caas-3BS.1 & TaFba-3B & TraesCS3B01G025200 & Fructose-bisphosphate aldolase \\
& TaBff-3B & TraesCS3B01G028700 & B-fructofuranosidase \\
QDek.caas-3BS.2 & TaABA4-3B & TraesCS3B01G039800 & Abscisic acid-deficient 4 \\
QDek.caas-4AL & TaSus-4A & TraesCS4A01G446700 & Sucrose synthase \\
\hline
\end{tabular}


(https://wheat.pw.usda.gov/ggpages/topics/Wheat660_ SNP_array_developed_by_CAAS.pdf), was used to test two isogenic lines BL31 and BL33 at CapitalBio Corporation (Beijing, China; http://www.capitalbio.com/). The frequency distribution of polymorphic SNPs in each chromosome was analyzed using Microsoft Excel 2016 (https://www.microsoft.com/zh-cn/). The target chromosomes with potential QTL were determined based on the frequency distribution of polymorphic SNPs in each chromosome.

\section{Genotyping by SSR markers}

Polymerase chain reaction (PCR) for SSR markers was performed in a reaction mixture of $15 \mu \mathrm{l}$ containing $7.5 \mu \mathrm{l}$ of $2 \times$ Taq PCR Mix (Beijing HT-biotech Co., Ltd., Beijing; http://www.ht-biotech.net/), $5 \mathrm{pmol}$ of each primer and $50-100 \mathrm{ng}$ of genomic DNA using the ABI Applied Biosystems Veriti 96 Well Thermal Cycler (Gene Co., Ltd., Shanghai; http://www.genecompany.com/). PCR amplification was performed at $95^{\circ} \mathrm{C}$ for $5 \mathrm{~min}$, followed by 35 cycles $\left(94{ }^{\circ} \mathrm{C}\right.$ for $30 \mathrm{~s}, 55-60^{\circ} \mathrm{C}$ for 30 s and $72^{\circ} \mathrm{C}$ for $\left.30 \mathrm{~s}\right)$ and a final extension at $72{ }^{\circ} \mathrm{C}$ for $7 \mathrm{~min}$. The PCR products were separated in $8 \%$ polyacrylamide gels at $250 \mathrm{~V}$ using the JY 600HE Electrophoretic Apparatus (Beijing Junyi Electrophoresis Co., Ltd., Beijing; http://www.bjjunyi.com/ ) or $2 \%$ agarose gels at $220 \mathrm{~V}$ using the DYY-7C Electrophoresis Apparatus (Beijing Liuyi Biotechnology Co., Ltd.; http://www.ly.com.cn/).

\section{Converting chip-based polymorphic SNPs into CAPS markers}

To enrich the target genetic region, polymorphic SNPs between BL31 and BL33, are needed to convert into flexible markers, such as kompetitive allele specific PCR (KASP) and CAPS markers. CAPS technology is an efficient tool to genotype SNPs [60], combining PCR with restriction enzymes digestion to differentiate the target alleles with polymorphic SNPs. The polymorphic SNPs between parents in the target region were identified by alignments with DNAMAN software (https://www.lynnon.com/index. html) for designing chromosome-specific CAPS markers [61]. The primers and restriction enzymes used for CAPS markers were listed in Additional file 4.

\section{Statistical analysis}

The analysis of variance (ANOVA) was conducted using the SAS 9.2 software (http://www.sas.com) with PROC MIXED procedure. Broad-sense heritability $\left(h_{\mathrm{B}}{ }^{2}\right)$ was calculated using the following formula [62]: ${h_{\mathrm{B}}}^{2}=\sigma_{\mathrm{g}}^{2} /$ $\left(\sigma_{\mathrm{g}}^{2}+\sigma_{\mathrm{ge}}^{2} / r+\sigma_{\varepsilon}^{2} / r e\right)$.

in which, $\sigma_{\mathrm{g}}^{2}, \sigma_{\mathrm{ge}}^{2}$ and $\sigma_{\varepsilon}^{2}$ were estimates of genotypes, genotype $\times$ environment interaction and residual error variances, respectively; $r$ and $e$ were replicates per environment and the number of environments, respectively.
The correlation coefficients of traits among three different environments were computed using the PROC CORR procedure with the SAS 9.2 software.

\section{Linkage map construction and QTL analysis}

Joinmap 4.0 software (http://www.kyazma.nl) was used for linkage map construction [63] and genetic distances between markers were estimated based on the Kosambi mapping function [64]. QTL analysis was conducted by inclusive composite interval mapping (ICIM) based on the Dek rate using IciMapping 4.0 software (http://www. isbreeding.net) $[65,66]$. The logarithm of odds (LOD) threshold score of 2.5 was determined for declaring significant QTL based on 1000 permutations with a type I error of $P<0.05$. The phenotypic variance explained (PVE) were calculated through stepwise regression to estimate individual QTL effects.

\section{Assays for grain starch and sucrose contents}

Six mature grains were grinded to fine powder in a mortar. The resultant powder $(0.1 \mathrm{~g})$ was used to measure starch contents with Starch Assay Kit (Beijing Solarbio Science \& Technology Co., Ltd., Beijing, http://www.solarbio.net/). Then, the following formula was used to convert $\mathrm{mg} / \mathrm{g}$ to $\mathrm{mg} /$ grain for scoring the grain starch content: Grain starch content $(\mathrm{mg} /$ grain $)=$ starch contents $(\mathrm{mg} / \mathrm{g}) * \mathrm{TGW} / 1000$.

TGW data was shown in Additional file 7. Six grains of two parents were collected at $5,10,15,20,25$, and 30 days after flowering (DAF) to validate the dynamic trend of grain sucrose contents during the filling stage. The dried grain were grinded to fine powder in mortar, and $0.1 \mathrm{~g}$ powder was used to measure grain sucrose contents using Plant Sucrose Assay kit (Beijing Solarbio Science \& Technology Co., Ltd., Beijing, http://www.solarbio.net/).

\section{Quantitative real-time PCR}

Total RNA was extracted from fresh grain 5, 15, and 25 days after flowering (DAF) using RNAprep pure Plant Kit (Tiangen Biotech Co., Ltd., http://www.tiangen. $\mathrm{com} /$ ). Reverse transcription was performed using the reverse transcriptase $\mathrm{M}-\mathrm{MLV}$ (RNase $\mathrm{H}^{-}$) (Takara Biomedical Technology Co., Ltd., http://www.takara.com). The iTaq ${ }^{\text {Tw }}$ Universal SYBR Green Supermix on CFX RealTime System (Bio-Rad) was used to perform quantitative real-time PCR (qPCR). The profile of $\mathrm{qPCR}$ was as follows: $95^{\circ} \mathrm{C}$ for $2 \mathrm{~min}$, followed by 40 cycles of $95^{\circ} \mathrm{C}$ for $5 \mathrm{~s}$ and $58^{\circ} \mathrm{C}$ for $20 \mathrm{~s}$. A final dissociation stage was run to generate a melting curve for judgement of amplification specificity. Quantification of qPCR was determined using the $2^{-\Delta \Delta \mathrm{Ct}}$ method [67]. Elongation factor $1 \alpha(E F 1 \alpha)$ gene was used as the internal control to calibrate the expression level of genes of interest. An additional file shows the primers used in qPCR (see Additional file 15). 


\section{Supplementary information}

Supplementary information accompanies this paper at https://doi.org/10. 1186/s12870-019-2102-6

Additional file 1: Figure S1. The phenotypes of BL31 (a) and BL33 (b) grains.

Additional file 2: Figure S2. The frequency distributions of polymorphic SNPs on 21 wheat chromosomes. The chromosomes with potential Dek QTL are highlighted in orange. The frequency threshold of 0.08 is indicated by a horizontal line. Distribution frequency is the percentage of polymorphic SNPs in the total ones on each chromosome.

Additional file 3: Table S1. SSR markers used in this study

Additional file 4: Table S2. SNPs used to develop CAPS markers.

Additional file 5: Figure S3. Design of CAPS marker for AX-109027972 (a), AX-108996126 (b), AX-109301653 (c), AX-109516011 (d) and AX110042240 (e). The arrows and boxes show the target sites and the positions of restriction sites for the CAPS markers, respectively. The underlined forward and reverse primers for CASP markers are labeled at specific target sites.

Additional file 6: Figure S4. PCR profile of molecular markers to enrich the target regions of QTL. (a) AX-109027972. (b) AX-108996126. (c) AX109301653. (d) AX-109516011. (e) AX-110042240. M, Marker (DL2000DNA ladder in a, b, c and e; 20 bp DNA ladder in d; Takara Bio Company). Target fragments are shown with red arrows. Lanes 1 and 2 indicate BL33 and BL31, respectively; lane 3 shows $F_{1}$ plants; lanes 4-10 are $F_{2}$ individual plants.

Additional file 7: Table S3. Comparison of TGW between BL31 and BL33.

Additional file 8: Figure S5. $L O D$ contours obtained by inclusive composite interval mapping (ICIM) analysis for TGW QTL on chromosomes $3 \mathrm{~B}$ in $\mathrm{F}_{2}$ and $\mathrm{F}_{2: 3}$ populations derived from BL33/BL31. The genetic region of QTGW.caas-3BS is highlighted in red. LOD contour in Gaoyi 2016-2017 cropping season and Gaoyi 2017-2018 cropping season indicated with red and green lines, respectively. LOD threshold of 2.5 is indicated by the dotted vertical line.

Additional file 9: Figure S6. The comparison of grain starch contents in mature grains between BL31 and BL33.

Additional file 10: Figure S7. The change of grain sucrose contents after flowering.

Additional file 11: Table S4. Annotated genes in the physical intervals of QTL.

Additional file 12: Table S5. Transcriptional patterns of candidate genes in the developing grains.

Additional file 13: Figure S8. Transcriptional patterns of four candidate genes in the developing grains.

Additional file 14: Figure S9. $P C R$ profile of the $W x-B 1$ specific marker M, Marker (DL2000DNA ladder; Takara Bio Company). Target fragments are shown with red arrows. Lane 1 indicates Chinese Spring; lane 2 indicates Guandong107; lane 3 and 4 show BL33 and BL31, respectively.

Additional file 15: Table S6. Primers used in quantitative real-time PCR.

\section{Abbreviations}

AGPP: adenosine diphosphate glucose pyrophosphorylase; ANOVA: analysis of variance; CAPS: cleaved amplified polymorphic sequence; CWI: cell wall invertase; DAF: days after flowering; Dek: defective kernel; Fba: fructosebisphosphate aldolase; GFD: grain filling duration; GFR: grain filling rate; GWAS: genome-wide association study; ICIM: inclusive composite interval mapping; IWGSC: International Wheat Genome Sequence Consortium; KASP: kompetitive allele specific polymerase chain reaction; LOD: logarithm of odds; PVE: phenotype variation explained; GPCR: quantitative real-time PCR; SNP: single nucleotide polymorphism; SSR: simple sequence repeat; Sus: sucrose synthase; TGW: thousand grain weight; WSC: water soluble carbohydrates

\section{Acknowledgments}

The authors are grateful to Prof. R. A. McIntosh, at Plant Breeding Institute, University of Sydney, for critical review of this manuscript.

\section{Authors' contributions}

$F C, T X L, F L P, X D A, W Y$ and $X X T$ performed the experiments and data analysis. DJY created and provided DEK lines for the research. CSH and ZY designed the experiments. FC and $\mathrm{CSH}$ wrote the draft. XXC and $\mathrm{HZH}$ revised the manuscript. All authors read and approved the final manuscript.

\section{Funding}

This work was funded by the National Key Research and Development Programs of China (2016YFD0100502), National Natural Science Foundation of China (31571663) and CAAS Science and Technology Innovation Program. The planting and phenotyping of materials were funded by National Natural Science Foundation of China (31571663) and CAAS Science and Technology Innovation Program, whereas the genotyping, data analysis and manuscript writing were funded by National Key Research and Development Programs of China (2016YFD0100502).

\section{Availability of data and materials}

The data sets supporting the results of this article are included within the article and its additional files.

Ethics approval and consent to participate

Not applicable.

\section{Consent for publication}

Not applicable.

\section{Competing interests}

The authors declare that they have no competing interests.

\section{Author details}

'Institute of Crop Sciences, National Wheat Improvement Center, Chinese Academy of Agricultural Sciences, Beijing 100081, China. ${ }^{2}$ Wheat Research Institute, Gansu Academy of Agricultural Sciences, Lanzhou 730070, China. ${ }^{3}$ International Maize and Wheat Improvement Center, 12 Zhongguancun South Street, Beijing 100081, China.

Received: 8 July 2019 Accepted: 28 October 2019

Published online: 08 November 2019

\section{References}

1. Cheng SH, Yang SM, Zhang BQ, Ji KZ, Zhao BH, Gao DR. Kernel plumpness and filling index in wheat (Triticum aestivum L.). Jiangsu J Agric Sci. 1993:9:7-10

2. Li FJ, Wen WE, He ZH, Liu JD, Jin H, Cao SH, et al. Genome-wide linkage mapping of yield-related traits in three Chinese bread wheat populations using high-density SNP markers. Theor Appl Genet. 2018;131:1903-24.

3. Neuffer MG, Sheridan WF. Defective kernel mutants of maize. I. Genetic and lethality studies. Genetics. 1980;95:929-44.

4. Lid SE, Gruis D, Jung R, Lorentzen JA, Ananiev E, Chamberlin M, et al. The defective kernel 1 (dek1) gene required for aleurone cell development in the endosperm of maize grains encodes a membrane protein of the calpain gene superfamily. Proc Natl Acad Sci. 2002;99:5460-5.

5. Chen XZ, Feng F, Qi WW, Xu LM, Yao DS, Wang Q, et al. Dek35 encodes a PPR protein that affects cis-splicing of mitochondrial nad4 intron 1 and seed development in maize. Mol Plant. 2017;10:427-41.

6. Garcia N, Li YB, Dooner HK, Messing J. Maize defective kernel mutant generated by insertion of a ds element in a gene encoding a highly conserved TTI2 cochaperone. Proc Natl Acad Sci. 2017;114:5165-70.

7. Li XJ, Wei G, Sun SL, Chen ZL, Jing C, Song W, et al. Defective Kernel 39 encodes a PPR protein required for seed development in maize. J Integr Plant Biol. 2018:60:45-64.

8. Röder MS, Korzun V, Wendehake $K$, Plaschke J, Tixier MH, Leroy P, et al. A microsatellite map of wheat. Genetics. 1998;149:2007-23.

9. Wang Y, Yang C, Jin QJ, Zhou DJ, Wang SS, Yu YJ, et al. Genome-wide distribution comparative and composition analysis of the SSRs in Poaceae. BMC Genet. 2015;16:18. 
10. UPOV-BMT, BMT/36/10 Progress report of the 36th session of the technical committee, the technical working parties and working group on biochemical and molecular techniques and DNA-profiling in particular, Geneva. 2002.

11. Sukumaran S, Dreisigacker S, Lopes M, Chavez P, Reynolds MP. Genomewide association study for grain yield and related traits in an elite spring wheat population grown in temperate irrigated environments. Theor Appl Genet. 2015;128:353-63.

12. Gao LL, Turner MK, Chao S, Kolmer J, Anderson JA. Genome wide association study of seedling and adult plant leaf rust resistance in elite spring wheat breeding lines. PLoS One. 2016;11:e0148671.

13. Kassa MT, You FM, Hiebert CW, Pozniak CJ, Fobert PR, Sharpe AG. Highly predictive SNP markers for efficient selection of the wheat leaf rust resistance gene Lr16. BMC Plant Biol. 2017;17:45.

14. Mourad AMI, Sallam A, Belamkar V, Wegulo S, Bowden R, Jin Y. Genomewide association study for identification and validation of novel SNP markers for Sr6 stem rust resistance gene in bread wheat. Front Plant Sci. 2018;9:380.

15. IWGSC, Appels R, Eversole K, Feuillet C, Keller B, Rogers J, et al. Shifting the limits in wheat research and breeding using a fully annotated reference genome. Science. 2018;361.

16. Cao SH, Guo XL, Liu DC, Zhang XQ, Zhang AM. Preliminary gene-mapping of photoperiod-temperature sensitive genic male sterility in wheat (Triticum aestivum L.). Acta Genet Sin. 2004;31:293-8.

17. Song QJ, Fickus EW, Cregan PB. Characterization of trinucleotide SSR motifs in wheat. Theor Appl Genet. 2002;104:286-93.

18. Guyomarc'h H, Sourdille P, Edwards K, Bernard M. Studies of the transferability of microsatellites derived from Triticum tauschii to hexaploid wheat and to diploid related species using amplification, hybridization and sequence comparisons. Theor Appl Genet. 2002;105:736-44.

19. Sourdille P, Tavaud M, Charmet G, Bernard M. Transferability of wheat microsatellites to diploid Triticeae species carrying the $\mathrm{a}, \mathrm{B}$ and D genomes. Theor Appl Genet. 2001;103:346-52.

20. Guyomarc'h H, Sourdille P, Charmet G, Edwards K, Bernard M. Characterisation of polymorphic microsatellite markers from Aegilops tauschii and transferability to the D-genome of bread wheat. Theor Appl Genet. 2002;104:1164-72.

21. Pestsova E, Ganal EW, Röder MS. Isolation and mapping of microsatellite markers specific for the D genome of bread wheat. Genome. 2000;43:689-97.

22. Sourdille P, Singh S, Cadalen T, Brown-Guedira GL, Gay G, Qi LL, et al. Microsatellite-based deletion bin system for the establishment of geneticphysical map relationships in wheat (Triticum aestivum L.). Funct Integr Genomics. 2004:4:12-25.

23. Somers DJ, Isaac P, Edwards K. A high-density microsatellite consensus map for bread wheat (Triticum aestivum L.). Theor Appl Genet. 2004;109:1105-14.

24. Zadoks JC, Chang TT, Konzak CF. A decimal code for the growth stages of cereals. Weed Res. 1974:14:415-21.

25. IWGSC. A chromosome-based draft sequence of the hexaploid bread wheat (Triticum aestivum) genome. Science. 2014;345:1251788.

26. Avni R, Nave M, Barad O, Baruch K, Twardziok SO, Gundlach H, et al. Wild emmer genome architecture and diversity elucidate wheat evolution and domestication. Science. 2017;357:93-7.

27. Luo MC, Gu YQ, Puiu D, Wang H, Twardziok SO, Deal KR, et al. Genome sequence of the progenitor of the wheat $\mathrm{D}$ genome Aegilops tauschii. Nature. 2017;551:498-502.

28. Wang SC, Wong D, Forrest K, Allen A, Chao S, Huang BE. Characterization of polyploid wheat genomic diversity using a high-density 90,000 single nucleotide polymorphism array. Plant Biotechnol. 2014;12:787-96.

29. Winfield MO, Allen AM, Burridge AJ, Barker GLA, Benbow HR, Wilkinson PA, et al. High-density SNP genotyping array for hexaploid wheat and its secondary and tertiary gene pool. Plant Biotechnol. 2016;14:1195-206.

30. Allen AM, Winfield MO, Burridge AJ, Downie RC, Benbow HR, Barker GL, et al. Characterization of a wheat breeders' Array suitable for highthroughput SNP genotyping of global accessions of hexaploid bread wheat (Triticum aestivum). Plant Biotechnol J. 2017;15:390-401.

31. Jin H, Wen WE, Liu JD, Zhai SN, Zhang Y, Yan J, et al. Genome-wide QTL mapping for wheat processing quality parameters in a Gaocheng 8901/Zhoumai 16 recombinant inbred line population. Front Plant Sci. 2016;7:1032S

32. Cui F, Zhang $N$, Fan $X L$, Zhang $W$, Zhao $C H$, Yang $L$, Pan $R$, Mei $C$, Jie $H$ Zhao X. Utilization of a Wheat660K SNP array-derived high-density genetic map for high-resolution mapping of a major QTL for kernel number. Sci Rep. 2017;7:3788.

33. Tian $X L$, Wen $W E$, Xie L, Fu LP, Xu DA, Fu C, et al. Molecular mapping of reduced plant height gene Rht24 in bread wheat. Front Plant Sci. 2017:8:1379.

34. Liu JD, He ZH, Wu L, Bai B, Wen WE, Xie CJ, et al. Genome-wide linkage mapping of QTL for black point reaction in bread wheat (Triticum aestivum L.). Theor Appl Genet. 2016;129:1-12.

35. Wang LX, Qiu J, Chang LF, Liu LH, Li HB, Pang BS, et al. Assessment of wheat variety distinctness using SSR markers. J Integr Agric. 2015;14: 1923-35.

36. Ramirez-Gonzalez RH, Segovia V, Bird N, Fenwick P, Holdgate S, Berry S, et al. RNA-Seq bulked segregant analysis enables the identification of highresolution genetic markers for breeding in hexaploid wheat. Plant Biotechnol J. 2015:13:613-24

37. Wang RX, Zhang XY, Wu L, Wang R, Hai L, Yan CS, et al. QTL mapping for grain filling rate and thousand-grain weight in different ecological environments in wheat. Acta Agron Sin. 2008;34:1750-6.

38. Groos C, Robert N, Bervas E, Charmet $\mathrm{G}$. Genetic analysis of grain proteincontent, grain yield and thousand-kernel weight in bread wheat. Theor Appl Genet. 2003:106:1032-40.

39. Hu YJ, Li MF, Yang DL, Liu Y, Cheng HB, Chang L, et al. Integration and meta-analysis of quantitative trait loci for water-soluble carbohydrate content in wheat grain. J Triticeae Crops. 2016;36:989-95.

40. Araki E, Miura H, Sawada S. Identification of genetic loci affecting amylose content and agronomic traits on chromosome 4A of wheat. Theor Appl Genet. 1999;98:977-84

41. Mccartney CA, Somers DJ, Lukow O, Ames N, Noll J, Cloutier S, et al. QTL analysis of quality traits in the spring wheat cross RL4452 × 'AC Domain'. Plant Breed. 2006:125:565-75.

42. Tian B, Liu B, Zhu ZL, Xie QG, Tian JC. Conditional and unconditional QTL mapping of grain starch accumulation in wheat. Sci Agric Sin. 2011:44:4551-9.

43. Wang JS, Liu WH, Wang H, Li LH, Wu J, Yang XM, et al. QTL mapping of yield-related traits in the wheat germplasm 3228. Euphytica. 2011; 177:277-92.

44. Cui F, Zhao CH, Ding AM, Li J, Wang L, Li XF, et al. Construction of an integrative linkage map and QTL mapping of grain yield-related traits using three related wheat RIL populations. Theor Appl Genet. 2014;127:659-75.

45. Tian B, Deng ZY, Xie QG, JC JT. Genetic dissection of the developmental behaviour of total starch content and its components in wheat grain. Crop Pasture Sci. 2015;66:445-55.

46. Deng ZY, Hu SN, Chen F, Li WJ, Chen JS, Sun CL, et al. Genetic dissection of interaction between wheat protein and starch using three mapping populations. Mol Breed. 2015;35:12.

47. Deng ZY, Fang WQ, Guo X, Zhao XX, Guo H, Hu SN, et al. Genetic dissection of interactions between wheat flour starch and its components in two populations using two QTL mapping methods. Mol Breed. 2018;38:41.

48. Nakamura T, Vrinten P, Saito M, Konda M. Rapid classification of partial waxy wheats using PCR-based markers. Genome. 2002;45:1150-6.

49. Uematsu K, Suzuki N, Iwamae T, Inui M, Yukawa H. Increased fructose 1, 6bisphosphate aldolase in plastids enhances growth and photosynthesis of tobacco plants. J Exp Bot. 2012:68:3001-9.

50. Iwaki T, Wadano A, Yokota A, Himeno M. Aldolase-an important enzyme in controlling the ribulose 1,5-bisphosphate regeneration rate in photosynthesis. Plant Cell Physiol. 1991;32:1083-91.

51. Wang LJ. Research progress of sucrose invertase in higher plants. J Anhui Agric Sci. 2014;42:8108-11.

52. Liao JH, Li JM, Wu Y. Research progress in starch synthase of wheat. J Guizhou Agric Sci. 2014:42:18-22.

53. North HM, De Almeida A, Boutin JP, Frey A, To A, Botran L, et al. The Arabidopsis ABA-deficient mutant aba4 demonstrates that the major route for stress-induced $A B A$ accumulation is via neoxanthin isomers. Plant J. 2010;50:810-24.

54. Wang Q, Wen XG, Lu CM, Zhang QD. Photosynthetic functions of different senescing leaves in the canopy of super high-yield rice 'Hua-An3'. Acta Phytoecol Sin. 2004;28:39-46.

55. Paulsen $\mathrm{H}$. Chlorophyll a/b-binding proteins. Photochem Photobiol. 1995;62:367-82.

56. Chourey PS, Nelson OE. The enzymatic deficiency conditioned by the shrunken-1 mutations in maize. Biochem Genet. 1976;14:1041-55. 
57. Hou J, Jiang QY, Hao CY, Wang YQ, Zhang HN, Zhang XY. Global selection on sucrose synthase haplotypes during a century of wheat breeding. Plant Physiol. 2014;164:1918-29.

58. Jiang QY, Hou J, Hao CY, Wang LF, Ge HF, Dong YS, Zhang XY. The wheat (T. aestivum) sucrose synthase 2 gene (TaSus2) active in endosperm development is associated with yield traits. Funct Integr Genomics. 2011;11:49-61.

59. Guillemaut P, Laurence MD. Isolation of plant DNA: a fast, inexpensive, and reliable method. Plant Mol Biol Report. 1992;10:60-5.

60. Shahinnia F, Sayed-Tabatabaei BE. Conversion of barley SNPs into PCRbased markers using dCAPS method. Genet Mol Biol. 2009;32:564-7.

61. Thiel T, Kota R, Grosse I, Stein N, Graner A. SNP2CAPS: a SNP and INDEL analysis tool for CAPS marker development. Nucleic Acids Res. 2004;32:e5.

62. Nyquist WE, Baker RJ. Estimation of heritability and prediction of selection response in plant populations. Crit Rev Plant Sci. 1991;10:235-322.

63. Stam P. Construction of integrated genetic linkage maps by means of a new computer package: join map. Plant J. 1993;3:739-44.

64. Kosambi DD. The estimation of map distances from recombination values. Ann Eugenics. 1943;12:172-5.

65. Li HH, Ye GY, Wang JK. A modified algorithm for the improvement of composite interval mapping. Genetics. 2007;175:361-74.

66. Wang J. Inclusive composite interval mapping of quantitative trait genes. Acta Agron Sin. 2009:35:239-45.

67. Schmittgen TD, Livak KJ. Analyzing real-time PCR data by the comparative $\mathrm{C}_{\mathrm{T}}$ method. Nat Protoc. 2008;3:1101-8.

\section{Publisher's Note}

Springer Nature remains neutral with regard to jurisdictional claims in published maps and institutional affiliations.

Ready to submit your research? Choose BMC and benefit from:

- fast, convenient online submission

- thorough peer review by experienced researchers in your field

- rapid publication on acceptance

- support for research data, including large and complex data types

- gold Open Access which fosters wider collaboration and increased citations

- maximum visibility for your research: over $100 \mathrm{M}$ website views per year

At $\mathrm{BMC}$, research is always in progress.

Learn more biomedcentral.com/submissions 